\title{
A Cerebellar High-Grade Neuroepithelial Tumour with BCOR Alteration in a five-year-old Child A case report
}

"Abeer Al-Battashi, ${ }^{1}$ Zahra Al Hajri, ${ }^{2}$ Arie Perry, ${ }^{3}$ Hunaina Al-Kindi, ${ }^{2}$ Ibrahim Al-Ghaithi ${ }^{4}$

$$
\begin{aligned}
& \text { ورم مخيخي ظهاري عصبي ذو درجة عالية مع تغيير جين } \\
& \text { BCOR عند طفل عمره خمس سنوات } \\
& \text { تقرير حالة }
\end{aligned}
$$

عبير البطاشية، زهرة الحجرية، آري بيري، حنينة الكندية، إبراهيم الغيثي

ABSTRACT: New groups of high-grade neuroepithelial tumours (HGNET) have emerged from the reclassification of central nervous system (CNS) embryonal tumours that have recognised CNS HGNET with BCOR alteration (CNS HGNET-BCOR). We report a two-year, nine-month-old Omani boy who presented to the Royal Hospital, Muscat, Oman, in 2015 with subacute head tilting and neck pain. A well-defined cerebellar lesion was found and he was treated with standard chemoradiotherapy. After a relapse at the age of five years, molecular testing revealed a $B C O R$ alteration. He was treated with further surgery and high-dose chemotherapy; unfortunately, he relapsed and died three years after he was diagnosed.

Keywords: Neuroepithelial Tumor; Glioblastoma; Human BCOR Protein; Embryonal Tumors; Case Report; Oman.

الملخص: ظهرت مجموعات جديدة من الأورام الظهارية العصبية عالية الدرجة (HGNET) من خلال إعادة تصنيف الأورام الجينية للجهاز

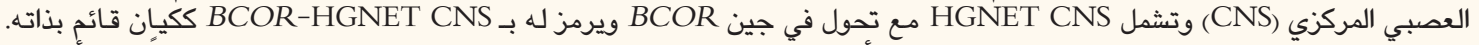

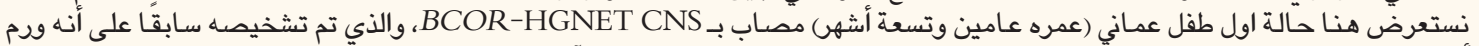

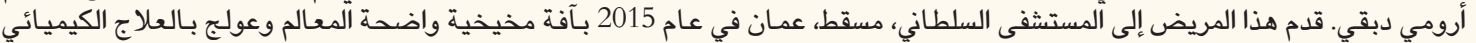

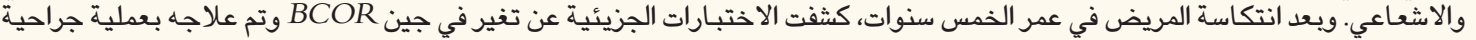

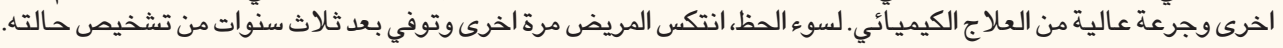

$$
\text { الكلمات المفتاحية: ورم ظهاري عصبي؛ ورم أرومي دبقي؛ بروتين BCOR البشري؛ أورام جنينية؛ تقرير حالة؛ عمان. }
$$

$\mathrm{M}$ ANAGING CHILDREN With CENTRAL nervous system (CNS) tumours is challenging as most data have been extrapolated from adult studies. ${ }^{1}$ Therefore, different diagnostic and therapeutic modalities have been implemented to tackle this challenge. Molecular testing using next-generation genome sequencing (NGS) has aided researchers in understanding the different behaviours and outcomes of tumours that are grouped by histopathology. In 2016, the World Health Organization (WHO) reclassified certain brain tumours according to their biology, for example, medulloblastoma and ependymoma. ${ }^{2}$ Further reclassification is being studied for other categories as tumours can be biologically distinct from each other, which delineates unique and separate tumour entities. This might aid in tailoring new treatment approaches to improve the survival of children affected by such tumours.

In 2016, Strum et al. described new brain tumour entities by reclassifying a group of central nervous system primitive neuroectodermal tumours (PNETs) using a DNA methylation technique and transcriptomic profiling. ${ }^{1}$ Very few cases have been described in the literature within this unique genetic landscape. ${ }^{1,3,4}$ This report describes the first Omani patient with $B C O R$ alteration in a CNS high-grade neuroepithelial tumour (HGNET).

\section{Case Report}

A two-year, nine-month-old boy presented in 2015 to the Royal Hospital, Muscat, Oman, with subacute head tilting and neck pain. In addition, he had a mild headache but no vomiting or altered sensorium. There was no significant personal or family history of tumours or malignancies. Clinically, he had a left side trochlear nerve palsy, resulting from high intracranial pressure and brainstem compression on the trochlear nucleus with no other focal neurological signs and no cerebellar signs. A magnetic resonance imaging (MRI) scan rev- 

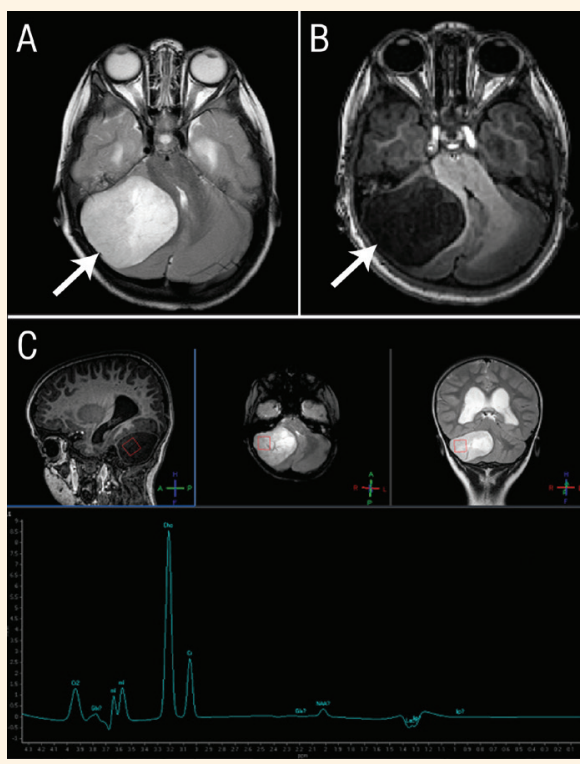

Figure 1: Magnetic resonance imaging scans of the head of a five-year-old boy showing a cerebellar primary tumour that is (A) hypointense on T1 and (B) hyperintense on T2 with both solid and cystic components. Magnetic resonance spectroscopy showed $(\mathbf{C})$ a choline peak with low $\mathrm{N}$-acetylaspartate and a lactate/lipid doublet.

ealed a right-sided cerebellar mass $(5.5 \times 5.6 \mathrm{~cm})$. It was well-defined, predominantly solid with some cystic components, hyperintense in $\mathrm{T} 2$ and hypointense in $\mathrm{T} 1$ with a mild diffusion restriction [Figures $1 \mathrm{~A}$ and $\mathrm{B}$ ]. The mass caused compression of the fourth ventricle with resultant hydrocephalus and a degree of tonsillar herniation. Magnetic resonance spectroscopy (MRS) showed a reduction in $\mathrm{N}$-acetylaspartate and a high choline peak with a lactate/lipid doublet that was indicative of a very aggressive tumour [Figure 1C]. There was no evidence of metastasis in the cerebral hemispheres, the spine or cerebrospinal fluid. These findings could be due to many aggressive infratentorial lesions, such as anaplastic ependymoma, high grade glioma, medulloblastoma and atypical teratoid rhabdoid tumour.

The patient underwent a gross total excision within a few days of his presentation using an occipital craniotomy approach. Intra-operatively, the tumour was very vascular. No residual tumour tissue was observed on the post-operative MRI scan. Pathology showed a well-circumscribed, highly cellular neoplasm composed of moderately pleomorphic cells with irregular nuclei and vesicular chromatin, arranged mostly in sheet-like to fascicular architectural patterns [Figure 2A]. In many areas, the tumour cells formed perivascular collections suggestive of pseudorosettes. Palisading necrosis and collections of apoptotic nuclei (apoptotic lakes) were also present and mitoses were abundant [Figures $2 \mathrm{~B}$ and $\mathrm{C}$ ]. Microvascular proliferation was not identified. Immunohistochemistry showed negative stains for glial fibrillary
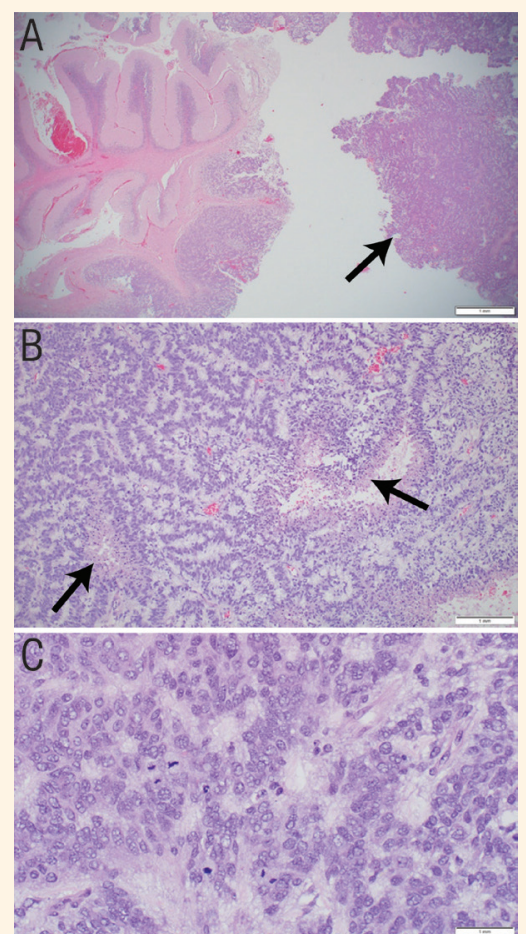

Figure 2: Haematoxylin and eosin stains (A) at x20 showing a highly cellular neoplasm (arrow) (B) at x100 magnification showing tumour cells arranged in a spongioblastic pattern and palisading necrosis with apoptotic lakes (arrows) and $(\mathbf{C})$ at x400 magnification showing numerous mitotic figures.

acidic protein, synaptophysin, and oligodendrocyte transcription factor 2 . The neuronal nuclei were focally positive for epithelial membrane antigen testing which demonstrated occasional cytoplasmic to paranuclear dot-like staining and the neurofilament protein staining had patchy positivity [Figure 3]. The tumour retained INI1 in the nuclei.

The patient was stable post-operatively and was started on bridging systemic chemotherapy, as he was less than three years of age at the time. The chemotherapy consisted of four cycles of vincristine, cyclophosphamide, cisplatin and etoposide. He was then initiated on chemoradiotherapy, during which he received focal radiotherapy in the form of intensity modulated radiotherapy at a total dose of 54 Gy in 30 fractions with concurrent temozolamide at a dose of $75 \mathrm{mg} / \mathrm{m}^{2}$. He was then started on maintenance temozolamide at a dose of $200 \mathrm{mg} / \mathrm{m}^{2}$; one year after presentation, he completed the twelve cycles uneventfully.

His post-radiotherapy MRI scan and his followup scans showed that he was in remission with no residual tumour tissue or recurrence. In 2017, at the age of five years and two months-exactly one year after the completion of treatment-a surveillance scan detected a local recurrence in the primary tumour bed in the right cerebellum (Figures $2 \mathrm{~B}$ and $4 \mathrm{~A}$ ). $\mathrm{He}$ underwent another gross total excision of the tumour with post-operative scans showing no residual tumour 

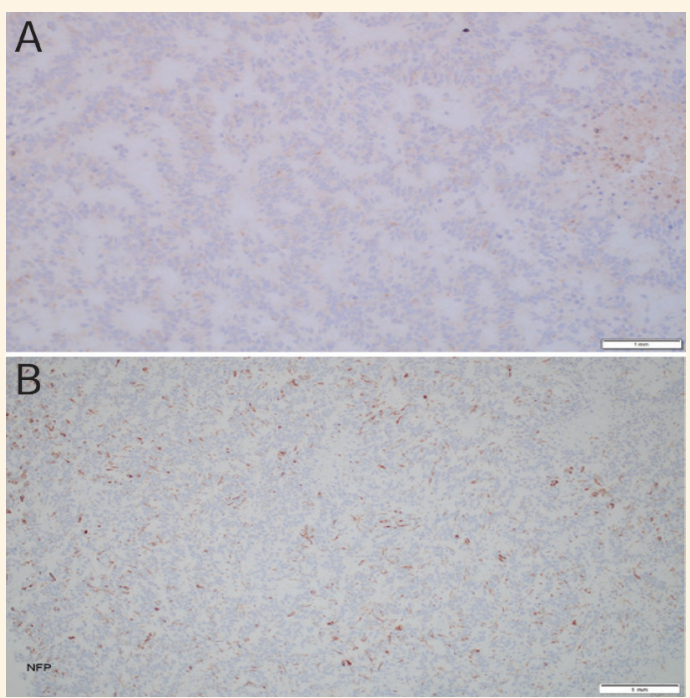

Figure 3: Immunohistochemistry of the tumour cells of a five-year-old boy. (A) Epithelial membrane antigen staining showing cytoplasmic to paranuclear dot-like staining and (B) sporadically positive neurofilament.

tissue. The histological features of the specimen were identical those of the specimen examined in 2015.

Next-generation sequencing (NGS) of the tumour was done using the University of California, San Francisco 500 Next-Generation Sequencing Cancer Gene Test that has the ability to analyse 479 cancer genes. It uses capture-based NGS by analysing genomic DNA from both tumour as well as normal cells. Target enrichment was performed by hybrid capture using custom oligonucleotides. It demonstrated an internal tandem duplication within exon 15 of the BCOR gene (this begins at codon p.V17141, which is an insertion of 30 amino acids). Additional alterations were telomerase reverse transcriptase (TERT) amplification and SMARCA2 mutation. Other alterations, such as IDH1, IDH2, Histone 3, MYCN, or PDGFRA were not demonstrated. The TERT and SMARCA2 alterations have not been previously reported within the CNSHGNET-BCOR entity.

The patient was restarted on temozolamide with the addition of bevacizumab (an antiangiogenic agent which inhibits the vascular endothelial growth factor). He received a total of six courses and remained well. Subsequently, he received four tandem autologous bone marrow transplants at the age of five years, six months based on the paediatric CNS embryonal tumour protocol. He presented with an extensive local relapse two months after transplantation. He developed severe obstructive hydrocephalus and reduced level of consciousness and was treated palliatively. He died three years after his initial presentation.

Verbal consent was obtained from the patient's parents for the publication of this case report.
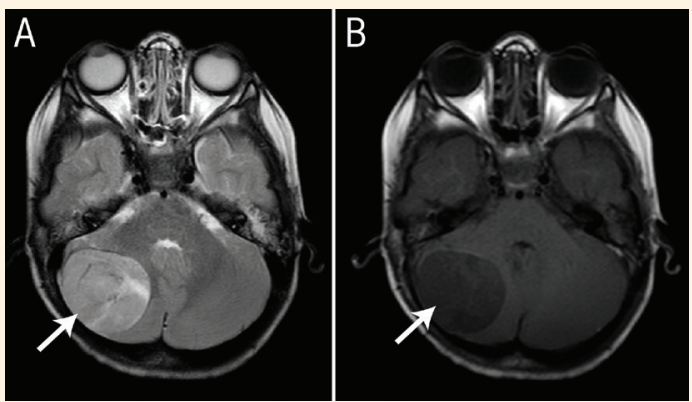

Figure 4: Magnetic resonance imaging scans of the head of a five-year-old boy showing the tumour at recurrence in the same primary site (arrows) with the same features as the primary tumour, as there is a well-circumscribed lesion that is (A) hypointense on T1 and (B) hyperintense on T2.

\section{Discussion}

A cluster of CNS PNETs, also known as embryonal tumours were regrouped into the following four molecular sub-categories by Strum et al. in 2016: CNS neuroblastoma with FOXR2 activation, CNS Ewing sarcoma family tumour with $C I C$ alteration, CNS high-grade neuroepithelial tumour with MN1 alteration and CNS HGNET-BCOR. ${ }^{1}$ There are very few reports of CNSHGNET-BCOR in the literature. This patient exhibited a common location for these tumours, which is within the cerebellum. However, these tumours can arise anywhere within the supratentorium or posterior fossa. ${ }^{1,3,4}$ This tumour has been rarely described as disseminating beyond the neuroaxis. There have been cases that described bone metastasis and subcutaneous nodule seeding.,

HGNET-BCOR was described after reviewing a variety of tumours, including medulloblastoma, ependymoma and high-grade glioma. ${ }^{1}$ Despite the different initial histological diagnosis, these tumours share pathological features including proliferation of uniform round or ovoid cells with fine chromatin and a rich arborising capillary network. Other characteristics may include stellate-, vacuolated- or spindle cell-cytomorphology, myxoid stromal changes and perivascular pseudorosettes. $^{3}$

Radiologically, HGNET-BCOR share features with other high-grade lesions including increased enhancement and diffusion restriction, as seen in the current patient. They can be solid, cystic or both and have features of a highly malignant lesion on MRS [Figure 1C]. The current patient had similar features to other patients described in the literature, with the mass being welldemarcated, hypointense on T1 and hyperintense on T2 [Figures 1A \& B and 4A \& B). ${ }^{1,2}$ The signal intensity in the present patient was fairly homogenous with a significant cystic component. 
Different treatments have been attempted for HGNET-BCOR. These included the use of multi-agents (cisplatin, etoposide and cyclophosphamide), bevacizumab, the use of intrathecal chemotherapy (methotrexate and topotecan) and autologous stem cell transplants. ${ }^{4}$ Yoshida et al. reported two patients who received autologous stem cell transplants and were both alive at the time of publication. ${ }^{4}$ In terms of local control, a total surgical excision is highly recommended and most patients received either focal radiotherapy or craniospinal radiotherapy with the majority receiving the former. ${ }^{3,4}$ In general, this tumour has a poor outcome and a tendency for relapse. ${ }^{3,4}$

\section{Conclusion}

The diagnostic approach to CNS tumours is evolving into sophisticated molecular profiling, which aids researchers in understanding the differences in behaviour between tumours that fall into the same histological classification and, therefore, their prognosis and different therapeutic approaches. The new WHO classification might reclassify these new entities into different categories. As the number of CNS HGNET-BCOR cases is very limited in the literature, more pathological, clinical and biological testing is required to understand this new entity.

\section{References}

1. Strum D, Orr BA, Toprak UH, Hovestadt V, Jones DTW, Capper D, et al. New brain tumor entities emerge from molecular classification of CNS-PNETs. Cell 2016; 164:1060-72. https://doi.org/10.1 016/j.cell.2016.01.015.

2. Louis DN, Perry A, Reifenberger G, von Deimling A, FigarellaBranger D, Cavenee WK, et al. The 2016 World Health Organization Classification of Tumors of the Central Nervous System: A summary. Acta Neuropathol 2016; 131:803-20. https://doi.org/10.1007/s00401-016-1545-1.

3. Appay R, Macagno N, Padovani L, Korshunov A, Kool M, André $\mathrm{N}$, et al. HGNET-BCOR Tumors of the cerebellum: Clinicopathologic and molecular characterization of 3 cases. Am J Surg Pathol 2017; 41:1254-60. https://doi.org/10.1097/ PAS.0000000000000866.

4. Yoshida Y, Nobusawa S, Nakata S, Nakada M, Arakawa Y, Mineharu Y, et al. CNS high-grade neuroepithelial tumor with BCOR internal tandem duplication: A comparison with its counterparts in the kidney and soft tissue. Brain Pathol 2018; 28:710-20. https://doi.org/10.1111/bpa.12585. 